\title{
Complication rates, lengths of stay, and readmission rates in "awake" and "asleep" deep brain simulation
}

\author{
Tsinsue Chen, MD, Zaman Mirzadeh, MD, PhD, Kristina Chapple, PhD, Margaret Lambert, BSN, RN, \\ and Francisco A. Ponce, MD
}

Department of Neurosurgery, Barrow Neurological Institute, St. Joseph's Hospital and Medical Center, Phoenix, Arizona

OBJECTIVE As the number of deep brain stimulation (DBS) procedures performed under general anesthesia ("asleep" DBS) increases, it is more important to assess the rates of adverse events, inpatient lengths of stay (LOS), and 30-day readmission rates in patients undergoing these procedures compared with those in patients undergoing traditional "awake" DBS without general anesthesia.

METHODS All patients in an institutional database who had undergone awake or asleep DBS procedures performed by a single surgeon between August 2011 and August 2014 were reviewed. Adverse events, inpatient LOS, and 30-day readmissions were analyzed.

RESULTS A total of 490 electrodes were placed in 284 patients, of whom 126 (44.4\%) underwent awake surgery and $158(55.6 \%)$ underwent asleep surgery. The most frequent overall complication for the cohort was postoperative mental status change (13 patients [4.6\%]), followed by hemorrhage (4 patients [1.4\%]), seizure (4 patients [1.4\%]), and hardwarerelated infection ( 3 patients [1.1\%]). Mean LOS for all 284 patients was $1.19 \pm 1.29$ days (awake: $1.06 \pm 0.46$ days; asleep: $1.30 \pm 1.67$ days; $p=0.08$ ). Overall, the 30 -day readmission rate was $1.4 \%$ ( 1 awake patient, 3 asleep patients). There were no significant differences in complications, LOS, and 30-day readmissions between awake and asleep groups.

CONCLUSIONS Both awake and asleep DBS can be performed safely with low complication rates. The authors found no significant differences between the 2 procedure groups in adverse events, inpatient LOS, and 30-day readmission rates.

https://thejns.org/doi/abs/10.3171/2016.6.JNS152946

KEY WORDS adverse events; asleep deep brain stimulation; complications; deep brain stimulation; functional neurosurgery; intraoperative imaging; length of stay; readmission

$\mathrm{D}$ EEP brain stimulation (DBS) is a well-established therapy for Parkinson's disease and essential tremor, with its safety and efficacy having been demonstrated in multiple clinical trials. . $^{4,17,22,23,36,37,44}$ Several studies have shown relatively low rates of overall mortality $(0 \%-8.3 \%)$, symptomatic intracranial hemorrhage $(0.8 \%-$ $5 \%$ ), and infection $(1.2 \%-15 \%)^{3,6,7,9,11,12,15,16,19,20,29,32,33,35,42,43}$ for DBS with microelectrode recording and intraoperative test stimulation. Traditionally, DBS has been performed with the patient "awake," without general anesthesia, using intraoperative test stimulation with or without microelectrode recordings to guide lead placement. In contrast, "asleep" DBS is performed using either direct targeting (for the globus pallidus interna [GPi] and subthalamic nucleus $[\mathrm{STN}])^{5,26,40}$ or indirect targeting (for the ventralis intermedius nucleus [VIM]), ${ }^{8}$ with the patient under general anesthesia and without intraoperative test stimulation. With improvements in MRI resolution and the accessibility of intraoperative imaging, asleep DBS surgery has gained popularity in the past several years. . $^{1,821,30,34}$

As published reports on functional outcomes of asleep DBS increase, $, 8,8,27,30,31,39$ the question of whether there is a difference in how patients tolerate the procedure becomes pertinent. In particular, factors such as extended time under general anesthesia may influence perioperative outcomes in this population. However, no large studies have reported complication rates for asleep DBS, and relatively few series have analyzed length of stay (LOS) and readmission

ABBREVIATIONS DBS = deep brain stimulation; GPi = globus pallidus interna; iCT = intraoperative CT; LOS = length of stay; MRSA = methicillin-resistant Staphylococcus aureus; PDQ-39 = 39-item Parkinson's Disease Questionnaire; STN = subthalamic nucleus; VIM = ventralis intermedius nucleus.

SUBMITTED December 16, 2015. ACCEPTED June 22, 2016.

INCLUDE WHEN CITING Published online September 23, 2016; DOI: 10.3171/2016.6.JNS152946. 
rates. As surgical techniques continuously evolve in an effort to improve the comfort, safety, and accessibility of the DBS operation, it becomes increasingly important to evaluate the impact of technical changes on perioperative safety, complication risk, and the duration of inpatient hospitalization. To address these issues, we retrospectively reviewed prospectively collected data on the adverse events, inpatient LOS, and 30-day readmissions for consecutive patients who had undergone either awake or asleep DBS surgery performed by a single neurosurgeon (F.A.P.).

\section{Methods}

Data were reviewed for all patients who had undergone either awake or asleep DBS surgery for movement disorders over a 3-year period (August 23, 2011-August 31, 2014). Targets were restricted to GPi, STN, and VIM (excluding one patient who underwent posterior subthalamic area DBS lead placement for essential tremor). Referring neurologists made all the primary diagnoses, and DBS candidacy was evaluated in a multidisciplinary consensus meeting of movement disorders neurologists, neurosurgeons, and neuropsychologists, at which targeting and laterality were also discussed. Both patient preference and the referring neurologist's approval determined whether DBS was performed in the awake or asleep state. Informed consent was obtained that emphasized that asleep DBS does not involve intraoperative test stimulation and therefore constitutes off-label use since successful intraoperative test stimulation is a prerequisite in the US Food and Drug Administration labeling for DBS. All patients were followed up via a prospectively maintained database established for DBS patients of the senior author (F.A.P.). This database was approved by the Institutional Review Board at St. Joseph's Hospital and Medical Center in Phoenix, Arizona. The most recent medical records of the patients were retrospectively reviewed to identify any additional complications not captured in the DBS database. All patients had a minimum of 6 months of follow-up.

Demographics and postoperative complications were reviewed. The total number of complications was analyzed based on the percentage of total leads. Hardware-related complications included infection, erosion, and revisions necessary for high impedance and lead malposition. Procedure-related complications included intraoperative and postoperative hemorrhage and seizure, cerebrospinal fluid leak, mental status change, pneumonia, and postoperative cerebral infarction. Inpatient LOS and 30-day readmission rates were also analyzed. For patients with multiple reasons for increased LOS, the primary etiology was noted.

\section{Surgical Procedure}

All procedures were performed utilizing uniform methods in each group (awake vs asleep). For all patients, preoperative imaging for direct (GPi or STN) or indirect (VIM) targeting was performed on a Siemens or General Electric 3-T MRI unit. DBS was performed with the patient either under intravenous sedation utilizing intraoperative test stimulation with or without microelectrode recordings ("awake") or under general anesthesia without mi- croelectrode recording or intraoperative test stimulation ("asleep").

For awake patients, all leads were implanted using the Leksell (Elekta AB) stereotactic frame with microelectrode recordings to guide GPi and STN lead placement, and intraoperative test stimulation was performed to assess for clinical benefit and appropriate thresholds for side effects. An intraoperative CT (iCT) scan was obtained postoperatively on a CereTom or BodyTom mobile scanner (NeuroLogica Corp.) to document stereotactic accuracy, and image coregistration with the preoperative MRI was performed using FrameLink software (Medtronic, plc). Bilateral electrodes were typically placed in one operation.

Patients in the asleep group had the stereotactic Leksell frame or NexFrame (Medtronic, plc) bone fiducials placed in the operating room after the induction of general anesthesia. After DBS leads were implanted, an iCT scan was obtained before skin closure to assess accuracy and to determine the necessity for lead repositioning. If a lead was repositioned, an additional iCT scan was obtained to confirm accuracy.

Pulse generators were placed either on the same day as lead implantation or in a separate operation 1 week later. (In January 2014, our institution transitioned primarily to an "all-in-one" approach with the pulse generator and DBS leads implanted on the same day, rather than the traditional practice [in the US] of implanting the pulse generator 1-2 weeks after lead implantation.)

We follow a strict protocol for pulse generator placement in which the targeted operative time is less than 30 minutes, only the primary surgeon handles the hardware, and exposure of the hardware is kept to a minimum (the scrub technician does not open or touch the hardware, and the generator is opened immediately before placing it in the pocket; this protocol was initiated following a personal communication with P. Starr on September 7, 2012, regarding protocols at the University of California, San Francisco). The generator is placed via a $5-\mathrm{cm}$ incision 2 fingerbreadths beneath the clavicle. Both the retroauricular and the generator incisions are irrigated copiously with gentamicin before implantation, and vancomycin powder $(500 \mathrm{mg})$ is placed into the retroauricular incision before closure.

In addition, beginning in August 2012, all patients underwent screening for methicillin-resistant Staphylococcus aureus (MRSA) before implantation. Patients and their household members were screened for MRSA at 4 sites: nares, throat, axilla, and groin. Patients who were MRSA positive were instructed to shampoo and shower with Hibiclens (chlorhexidine gluconate; Mölyncke Health Care) daily for 2 weeks before surgery. Seven days before surgery, patients initiated the application of mupirocin calcium $2 \%$ nasal ointment to both nares twice daily. Thirty minutes before skin incision, vancomycin (500 mg) was administered intravenously.

\section{Statistical Analysis}

Frequencies with percentages and means with standard deviations were used to describe the overall study cohort and patients with and without complications. Independent- 
samples t-tests were used to compare mean differences for continuous variables, and chi-squared or Fisher's exact tests were used for ordinal or dichotomous variables. To account for familywise error, we adjusted the significance threshold by dividing the standard cutoff of 0.05 by the number of statistical tests (50 tests) and obtained an adjusted threshold value of 0.001 . Logistic regression models were then used to determine significant predictors of complications and mental status change, the most frequent complication. Because of the small sample size, patients with a dystonia diagnosis were excluded from regression analyses. We used SPSS Statistics for Windows, version 22 (IBM Corp.), for analyses.

\section{Results}

A total of 490 DBS electrodes were placed in 284 patients over a 36-month period. Demographics of the overall cohort and a comparison of the awake and asleep groups are shown in Table 1. Of the 284 patients undergoing implantation for DBS, 126 (44.4\%) underwent awake surgery and $158(55.6 \%)$ underwent asleep surgery. Pulse generators were placed either on the same day as lead implantation $(29.6 \%)$ or in a separate operation 1 week later (70.4\%). Mean follow-up was $21.4 \pm 9.8$ months (range 6-41 months). The most common indication for surgery was Parkinson's disease (71.1\%), followed by essential tremor $(26.0 \%)$ and dystonia $(2.8 \%)$.

\section{Awake Versus Asleep Group Demographics}

There were no differences in age or sex between the awake and asleep groups. There was a significantly greater proportion of bilateral cases in the asleep group (135 cases [85.4\%]) than in the awake group (71 cases [56.3\%]) (p $<0.001)$ and a greater proportion of staged cases in the awake group (102 cases [81.0\%]) than in the asleep group $(98[62.0 \%])(\mathrm{p}=0.001)$. Although the distribution of indications for surgery was significantly different between the asleep and awake groups $(\mathrm{p}<0.001)$, the 2 groups consisted predominantly of Parkinson's patients (awake $61.1 \%$, asleep $79.1 \%$ ), followed by essential tremor patients (awake 37.3\%, asleep 17.1\%) and dystonia patients (awake $1.6 \%$, asleep $3.8 \%$ ). The distribution of targets between the 2 groups was significantly different $(\mathrm{p}<0.001)$. The VIM was the most frequent target in the awake group (52 cases [41.3\%]), followed by the GPi (43 cases [34.1\%]) and STN (31 cases [24.6\%]). In the asleep group, the GPi was the most frequent target (94 cases [59.5\%]), followed by the STN (36 cases [22.8\%]) and VIM (28 cases [17.7\%]).

There were no significant differences in the rest of the demographic variables between or within the awake and asleep groups. There were no intraoperative deaths, seizures, or aborted procedures. There was 1 perioperative death: a 69-year-old man with multiple medical comorbidities, who had undergone awake, staged, unilateral VIM electrode placement for Parkinson's disease, died 2 months postoperatively as a result of gastrointestinal medical issues.

Subcategories of complications were compared between DBS techniques (awake vs asleep, staged vs allin-one), number of leads placed (unilateral vs bilateral),
TABLE 1. Patient demographics and DBS categorization*

\begin{tabular}{lcccc}
\hline \multicolumn{1}{c}{ Variable } & All Patients & $\begin{array}{c}\text { Awake } \\
\text { Cohort }\end{array}$ & $\begin{array}{c}\text { Asleep } \\
\text { Cohort }\end{array}$ & p Value \\
\hline No. of patients & 284 & 126 & 158 & \\
\hline Mean age in yrs & $64.0 \pm 10.1$ & $64.1 \pm 10.5$ & $63.9 \pm 9.8$ & 0.841 \\
\hline Sex & & & & 0.243 \\
\hline Male & $181(63.7)$ & $85(67.5)$ & $96(60.8)$ & \\
\hline Female & $103(36.3)$ & $41(32.5)$ & $62(39.2)$ & \\
\hline Lead & & & & $<0.001$ \\
\hline Unilat & $78(27.5)$ & $55(43.7)$ & $23(14.6)$ & \\
\hline Bilat & $206(72.5)$ & $71(56.3)$ & $135(85.4)$ & \\
\hline Target & & & & $<0.001$ \\
\hline GPi & $137(48.2)$ & $43(34.1)$ & $94(59.5)$ & \\
\hline STN & $67(23.6)$ & $31(24.6)$ & $36(22.8)$ & \\
\hline VIM & $80(28.2)$ & $52(41.3)$ & $28(17.7)$ & \\
\hline Indication & & & & $<0.001$ \\
\hline PD & $202(71.1)$ & $77(61.1)$ & $125(79.1)$ & \\
\hline Essential tremor & $74(26.1)$ & $47(37.3)$ & $27(17.1)$ & \\
\hline Dystonia & $8(2.8)$ & $2(1.6)$ & $6(3.8)$ & \\
\hline Surgery type & & & & 0.001 \\
\hline All-in-one & $84(29.6)$ & $24(19.0)$ & $60(38.0)$ & \\
\hline Staged & $200(70.4)$ & $102(81.0)$ & $98(62.0)$ & \\
\hline PD = Parkinson's disease. & & & \\
* Values represent number (percentage) or mean \pm standard deviation. & \\
\hline
\end{tabular}

and targets (GPi vs STN vs VIM) (Table 2). No patients experienced postoperative pneumonia, cerebrospinal fluid leak, or cerebral infarction (clinical or radiographic). Patients were returned to the operating room for infection, hardware failure, lead repositioning, and subdural hematoma due to a postoperative ground-level fall. Patients who experienced complications were significantly older than those who did not $(68.2 \pm 10.1$ vs $63.6 \pm 10.0$ years, $p=$ $0.03)$.

\section{Hardware-Related Complications}

The most common hardware-related complication was infection (3 patients [1.1\%] with 6 leads [1.2\%]). In all 3 cases, purulence developed at the pulse generator site only at the time of presentation. Wound cultures grew Pseudomonas aeruginosa and Proteus mirabilis (1 patient), coagulase-positive $S$. aureus and Propionibacterium acnes (1 patient), and MRSA (1 patient).

The second most frequent hardware complication was revision due to problems with impedance (2 patients [0.7\%] with 3 leads). One case was detected immediately postoperatively in the recovery room, after an intraoperative normal impedance check. The patient was taken back to the operating room, where the problem was localized to the extension wire. It was switched out, and there were no further complications. The second patient also had normal impedance intraoperatively; however, at the initial programming 20 days after implantation, abnormal impedance was found on all combinations involving Contacts 3 and 1, ranging from 10,000 to $16,000 \mathrm{ohms}$. The patient underwent open interrogation 30 days after initial implan- 


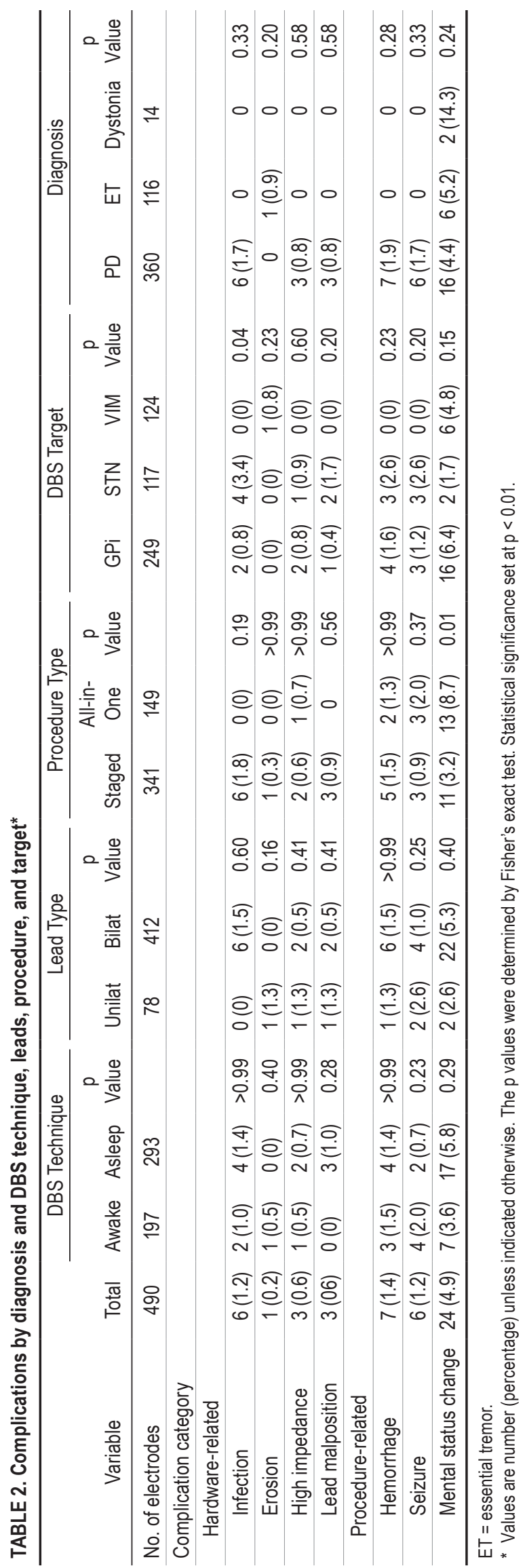

tation, and the extension wire was replaced. There were no further complications.

\section{Procedure-Related Complications}

The most common procedure-related complication was mental status change (13 patients [4.6\%] with 24 electrodes [4.9\%]), followed by postoperative hemorrhage (4 patients [1.4\%] with 7 electrodes [1.4\%]). Of the 4 patients with hemorrhage, 1 suffered an asymptomatic acute subdural hematoma seen immediately on postoperative $\mathrm{CT}$ and 3 had normal postoperative CTs but developed symptomatic hemorrhages within 7 days of surgery. One patient experienced a focal subarachnoid hemorrhage, 1 had bilateral subdural hygromas, and 1 had both an acute subdural hematoma and an intraparenchymal contusion. The patient with unilateral subdural hematoma eventually required surgical evacuation on postoperative Day 14 because of symptomatic progressive mass effect (Fig. 1) and returned to neurological baseline postoperatively. Four patients $(1.4 \%)$ with 6 electrodes $(1.2 \%)$ had generalized tonic-clonic seizures; none had a seizure history. None of the 4 had new intracranial pathology when they again underwent $\mathrm{CT}$ scanning after the seizure.

Overall, the mean age of the patients with mental status change (70.2 \pm 8.5 years) was not significantly higher than that of patients without such a change $(63.7 \pm 10.0$ years) $(\mathrm{p}=0.89)$. No statistically significant differences were found in categorical complication rates between awake and asleep, unilateral and bilateral, or staged and all-in-one groups or among the 3 DBS targets or 3 primary indications. There was, however, a somewhat higher percentage of patients in the all-in-one group who developed a change in mental status $(\mathrm{p}=0.02)$. Multivariate logistic regression analysis demonstrated no association between complications and sex, age, primary diagnosis, and DBS technique (awake vs asleep, unilateral vs bilateral, all-in-one vs staged). There was also no significant association among these variables with regard to mental status change, although patients who were 65 years old or older had an association trending toward significance (OR $4.71,95 \%$ CI $0.99-22.32, p=0.05$ ) (Table 3). In the subset of 13 patients who experienced a mental status change, 9 had undergone asleep placement. In the asleep group, a significantly higher proportion of patients with mental status change were 65 years old or older $(9[100 \%]$ of 9$)$ compared with those without mental status change (74 [49.7\%] of 149) $(\mathrm{p}=0.003)$.

\section{Parkinson's Disease Patients}

Among the entire study cohort, $71.1 \%$ (202) of patients had Parkinson's disease (38.1\% awake, $61.9 \%$ asleep). A separate analysis was performed of hardware- and procedure-related complications in this group. Among the Parkinson's patients, 10 patients $(5.0 \%)$ with 16 leads $(4.4 \%$ of 360 leads) experienced a mental status change, and more of these leads were placed using the all-in-one procedure (13 leads [11.2\%]) versus the staged procedure (3 leads $[1.2 \%])(p=0.009)$. No other differences were found among other technique variables (awake vs asleep, unilateral vs bilateral, GPi vs STN). Separate analyses for es- 


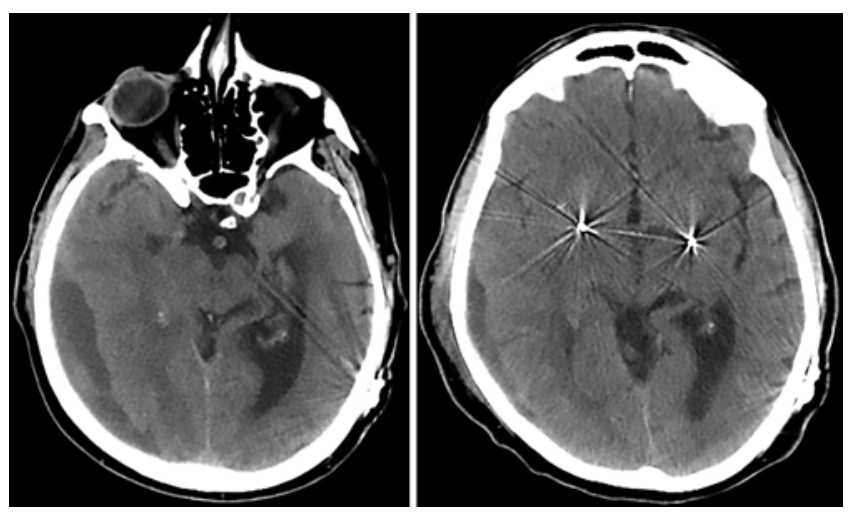

FIG. 1. Left: Axial CT scan obtained on postoperative Day 14, demonstrating increased size of right parietal subdural hematoma with mass effect. Right: Postoperative CT scan obtained after subdural hematoma evacuation.

sential tremor and dystonia groups could not be performed because of their smaller sample sizes.

\section{Length of Stay and Readmissions}

The mean LOS for all 284 patients was $1.19 \pm 1.29$ days (range 1-19 days, median 1 day); for asleep patients it was $1.30 \pm 1.67$ days (range 1-19 days, median 1 day), and for awake patients it was $1.06 \pm 0.46$ days (range 1-6 days, median 1 day) $(\mathrm{p}=0.08)$. At our institution, patients are typically admitted overnight and discharged the following morning, if appropriate. Sixteen patients (5.6\%) required a prolonged hospital stay ( $\geq 2$ nights) ranging from 2 to 19 days (mean $4.25 \pm 4.21$ days, median 3 days). The most common reasons for a prolonged inpatient stay were mental status change (4 cases), nausea/emesis (3 cases), and urinary retention (3 cases). Less common reasons were hemorrhage (2 cases) and oxygen desaturation, transient dysarthria, fever, and seizure (1 case each).

Four patients (1.4\%) were readmitted within 30 days of implantation; all had undergone asleep DBS surgery. One patient had a mechanical fall and presented with bilateral subdural hematomas requiring evacuation. This patient required a prolonged hospitalization (13 nights) because of challenges with insurance authorization for acute rehabilitation. Of the remaining 3 patients, 1 developed spontaneous headache secondary to atraumatic bilateral subdural hygromas, which were conservatively managed with 2 nights of observation. The second patient was readmitted for concerns about seizure activity; however, seizure workup was negative. This patient's symptoms spontaneously resolved, and he was discharged after a 4-night hospitalization. The third patient was readmitted for new-onset seizure and was discharged after 1 night of hospitalization. The mean LOS for this group of patients upon readmission was $5.0 \pm 5.8$ days.

In the patient groups with prolonged hospital stays $(\geq 2$ or $\geq 3$ nights) and 30-day readmissions, there were no statistically significant differences between asleep and awake groups, unilateral and bilateral leads, or staged and all-inone procedures, nor were there differences among DBS targets or DBS indications. More patients in the asleep group (13 [8.2\%]) than in the awake group (3 [2.4\%]) (p
TABLE 3. Logistic regression models predicting complications and mental status change

\begin{tabular}{|c|c|c|c|c|c|c|}
\hline \multirow[b]{2}{*}{ Variable } & \multicolumn{3}{|c|}{ Complications } & \multicolumn{3}{|c|}{ Mental Status Change } \\
\hline & $\begin{array}{c}p \\
\text { Value }\end{array}$ & OR & $95 \% \mathrm{Cl}$ & $\begin{array}{c}\mathrm{p} \\
\text { Value }\end{array}$ & OR & $95 \% \mathrm{Cl}$ \\
\hline Sex & 0.17 & 0.48 & $0.17-1.35$ & 0.21 & 0.36 & $0.07-1.75$ \\
\hline $\begin{array}{c}\text { Asleep (vs } \\
\text { awake) }\end{array}$ & 0.55 & 1.35 & $0.51-3.55$ & 0.33 & 2.08 & $0.48-9.08$ \\
\hline Unilat (vs bilat) & 0.52 & 0.72 & $0.26-1.98$ & 0.61 & 1.54 & $0.30-7.89$ \\
\hline PD (vs ET) & 0.33 & 0.56 & $0.18-1.79$ & 0.59 & 1.50 & $0.35-6.44$ \\
\hline $\begin{aligned} & \text { Age } \geq 65 \text { yrs } \\
& \text { (vs }<65 \text { yrs) }\end{aligned}$ & 0.16 & 1.90 & $0.78-4.65$ & 0.05 & 4.71 & $0.99-22.32$ \\
\hline $\begin{array}{l}\text { All-in-one (vs } \\
\text { staged) }\end{array}$ & 0.17 & 0.48 & $0.17-1.35$ & 0.10 & 2.88 & $0.81-10.25$ \\
\hline
\end{tabular}

$=0.04)$ had a hospital stay $\geq 2$ nights, and more patients in the asleep group (8 [5.1\%]) than in the awake group (1 [0.8\%]) $(\mathrm{p}=0.047)$ had a hospital stay $\geq 3$ nights, although these findings did not reach statistical significance for this analysis (Table 4).

\section{Discussion}

There is growing interest in performing DBS under general anesthesia. Our initial experience has demonstrated equivalent functional outcomes between awake and asleep groups. ${ }^{8}$ We found that essential tremor patients who underwent asleep VIM placement demonstrate no difference in the percentage of postoperative improvement, as measured by their Bain and Findley Tremor Activities of Daily Living scores $(48.6 \%),{ }^{8}$ compared with awake patients $(45.5 \%)(\mathrm{p}=0.35)$. Additionally, at 6-month follow-up, Parkinson's patients who had undergone asleep GPi placement had significant improvement in mean off-medication motor scores (48.4 vs 29.8, $\mathrm{p}<0.001)$ and in 39-item Parkinson's Disease Questionnaire (PDQ-39) scores (50.3 vs $42.0, \mathrm{p}=0.03$ ). Daily levodopa-equivalent doses were also significantly decreased at 6 months (1207 vs $1035 \mathrm{mg}, \mathrm{p}=$ 0.004) ${ }^{27}$ As reports of functional outcome data for asleep DBS have increased in the medical literature, the effect of anesthesia on perioperative recovery has become an area of growing concern for neurologists and patients. Complication rates and LOS data for a large series of patients who have undergone asleep DBS surgery under general anesthesia have not been reported. In this study, we found no difference in hardware- or procedure-related complications in patients undergoing asleep and awake surgeries. In addition, we found no difference in the mean LOS of these 2 groups.

\section{Complications}

Our overall hardware complication rate was $6.0 \%$, which is within the lower range reported for previous studies (4.9\%-22.8\%). ${ }_{3}^{3,6,7,9,15,16,19,20,29,32,33,35,42,43}$ Our most frequent hardware-related complication was infection (6 leads [1.2\%] in 3 patients [1.1\%]), and this rate is also within the lower range of previously published infection rates $(0.4 \%-15.2 \%)^{6,7,9,11,12,15,16,19,20,29,32,33,35,42,43}$ This wide range 


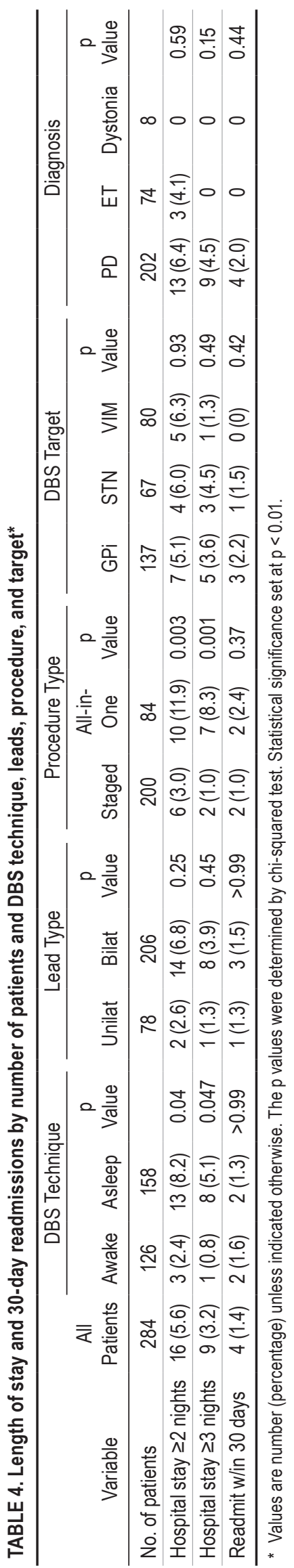

of reported infection rates includes some smaller series of $30-40$ patients. ${ }^{19,22,37}$ However, 2 recent large, single-center series have also demonstrated low infection rates of $4.5 \%$ in 759 leads placed in 420 patients $^{38}$ and of $1.18 \%$ in 510 leads placed in 392 patients. ${ }^{32}$

We believe that our low infection rate is primarily attributable to strict adherence to our pulse generator placement protocol, which includes restricting generator handling to one person, applying vancomycin powder to the cranial incision, and using a preoperative MRSA-screening and treatment protocol. We used the same generator protocol across all patients and found no difference in hardwarerelated complications in asleep versus awake groups, unilateral versus bilateral groups, or all-in-one versus staged groups, or among DBS target groups.

Mental status change was the most common procedure-related complication (24 leads [4.9\%] in 13 patients [4.6\%]), which is consistent with data in the literature, with reported rates of $4.7 \%-27.7 \%$ for unilateral procedures, $10.7 \%-14.6 \%$ for simultaneous bilateral procedures, and $3.5 \%-22 \%$ for staged bilateral procedures. ${ }^{32}$ The rate of mental status change in our patients with simultaneous bilateral implanted electrodes (5.4\%) is lower than historical rates $(10.7 \%-14.6 \%){ }^{16,19,32,43}$ This finding may be related, in part, to the incomplete capture of all patients experiencing a mental status change, because of the variability and subjectivity of symptoms within this category and the challenges faced when discerning symptoms from a chart review. It may also be related to the relative decrease in time required to place a second electrode (data not reported).

Hemorrhage occurred in 4 patients $(1.4 \%)$ with 7 leads $(1.4 \%)$. Three patients were symptomatic, and 1 of these 3 required additional craniotomy for subdural hematoma evacuation. This rate is within the range of previously reported rates for intracranial hemorrhages $(1.1 \%-6.29 \%)$ in studies with 30-420 patients. 3,6,7,9,15,16,19,20,29,32,33,35,42,43 Multivariate analysis showed no increased risk in overall complications associated with sex, increased age ( $\geq 65$ vs $<65$ years), diagnosis (Parkinson's disease vs essential tremor), or DBS technique (awake vs asleep, unilateral vs bilateral, all-in-one vs staged). However, further analysis of the asleep group showed that a significantly higher proportion of patients with than without mental status change were 65 years old or older. This finding is in line with established knowledge that older patients are more likely to experience mental status changes after general anesthesia, regardless of the surgical procedure. All mental status changes in our patients were temporary, and patients were discharged home at their neurological baseline.

Previous studies have also demonstrated a significant association between increasing age and hemorrhage, raising concern that older patients may have a higher complication rate and may not tolerate DBS surgery as well as younger patients..$^{45}$ However, 2 recent database analyses of 661 patients undergoing DBS for essential tremor and of 1757 patients undergoing DBS for Parkinson's disease with 90-day follow-up also demonstrated no increased risk of complications associated with increasing age from $<50$ to 90 years for either group. ${ }^{10,41}$ Further subanalysis of patients experiencing mental status change showed a trend toward significance for a positive association with those 65 
years old or older; however, a larger sample size is likely needed to detect true significance.

We performed a separate analysis among the Parkinson's patients (202 patients) to eliminate variability introduced by other disease groups (essential tremor and dystonia). Results showed that significantly more patients experienced mental status change in the all-in-one placement group versus the staged placement group $(\mathrm{p}=0.009)$. This finding may be related to the longer duration of continuous anesthesia for patients undergoing the all-in-one procedure.

\section{Length of Stay and Readmission Rates}

There are limited data on LOS and readmission rates after DBS. While most patients stay only 1 hospital night after DBS implantation, a prolonged LOS can occur for several reasons. In a recent series by Mikos et al., ${ }^{24} 115$ unilateral DBS electrodes were placed in Parkinson's patients, $21 \%$ of whom required a hospital stay of more than 1 night. The most common reason for an increased LOS was mental status change, followed by hemorrhage, with a total LOS ranging from 2 to 60 days. In Goodman et al.'s ${ }^{13}$ series of 191 electrodes in 100 patients, the mean LOS was 3.1 days; however, these patients were admitted 1 day before surgery to facilitate preoperative transitioning off Parkinson medications. Mental status change was also the most frequent cause of increased LOS in this group. In our series, only $5.6 \%$ of patients required a hospital stay of $\geq 2$ nights, and the mean LOS was $1.19 \pm 1.29$ days. Our patients are admitted on the day of surgery, rather than on the preceding day.

The leading cause of increased LOS in our patients was also mental status change (4 patients), followed by nausea and/or emesis (3 patients) and urinary retention (3 patients). All 3 patients with an increased LOS due to nausea and/or emesis and 2 of the 3 patients with urinary retention were in the asleep group. Although there was a trend toward more asleep patients with $\mathrm{a} \geq 2$-night hospital stay $(8.2 \%)$ than awake patients $(2.4 \%)(\mathrm{p}=0.04)$ and more asleep patients with $\mathrm{a} \geq 3$-night hospital stay (5.1\%) than awake patients $(0.8 \%)(\mathrm{p}=0.047)$, these findings did not reach statistical significance for this analysis. Given the standard threshold of $p<0.05$, the comparison between 13 patients (8.2\%) and 3 patients (2.4\%) would be considered statistically significant, but with only $53 \%$ power. To account for familywise error, we adjusted the threshold for our study to $\mathrm{p}<0.01$, and thus the association between hospital LOS $\geq 2$ and $\geq 3$ days with awake versus asleep implantation was not statistically significant. Setting alpha at 0.01 would require at least 200 patients in the awake group and 251 in the asleep group to detect a statistically significant difference between $8.2 \%$ and $2.4 \%$. All patients with a $\geq 3$-night hospital stay (9 patients [3.2\%]) had Parkinson's disease, although this finding was not statistically significant either. These trends may be related to factors associated with the postoperative side effects of general anesthesia. Multivariate analysis could not be performed because of the small sample size.

A recent study reviewing 30-day readmissions for cranial neurosurgical procedures within the categories of neoplasm, vascular disease, seizure, and trauma found readmission rates that ranged from $14 \%$ (seizure group) to $24 \%$ (vascular group). ${ }^{28}$ In a series of 303 patients from our institution, the 30-day readmission rate after transsphenoidal surgery for pituitary tumor was $8.9 \%$, with delayed hyponatremia (55.6\%) as the most frequent cause of readmission. ${ }^{4}$ Relatively few studies have evaluated readmission rates for DBS patients; however, a recent study by Jacob et al. ${ }^{18}$ found a 30 -day readmission rate of $4.3 \%$ for 211 DBS patients. In the current study, we found a comparably low 30 -day readmission rate of $1.4 \%$. The lower readmission rates for DBS patients compared with those for other neurosurgical patients probably reflect the nature of the pathology being treated and the increased severity of illness among patients with neoplastic, vascular, epileptic, and traumatic diseases. These neurosurgical pathologies often necessitate emergent or urgent surgical intervention, whereas DBS procedures are performed on an elective basis.

\section{Avoidance of Complications and Lessons Learned Infection}

The 3 patients with hardware-related infections required complete explantation despite attempts to salvage the DBS system. To avoid futile salvage attempts in the future, we therefore now explain to patients preoperatively that the definitive treatment for infection is complete system explantation. Interestingly, these 3 infections occurred early in both the awake experience (Case 1) and the asleep experience (Cases 4 and 7). This suggests that, with appropriate attention and experience, the rate of infection for DBS surgery can be kept to a minimum. After a MRSA infection occurred in a 52-year-old male patient, we instituted a MRSA-screening program. We also use vancomycin powder $(1000 \mathrm{mg})$ during lead placement for the frontal incisions, vancomycin powder $(500 \mathrm{mg})$ during battery placement for the retroauricular incision, and a TYRX (Medtronic, plc) antibacterial pouch for the generator, as well as the aforementioned protocol regarding surgery duration and hardware handling.

We believe that the MRSA-screening process is effective, because there were no additional MRSA infections after its initiation (August 2012) through the end of the study period (August 2014). However, other factors outlined in the pulse generator protocol, such as the use of vancomycin powder, may have also contributed to this finding. Overall, 225 patients were swabbed during the study period, and 6 patients $(2.67 \%)$ tested positive preoperatively and were treated. Four patients were colonized in the nares; 1 patient in the throat; and 1 patient in the nares, axilla, and throat.

On the basis of recommendations from our Infectious Disease Department after reviewing the initial MRSA detection rate, we modified our protocol (as of December 2015) to swab patients who answer "yes" to one of our screening questions: Have you or any of your family members been treated for MRSA? Have you or any of your family members worked in a health care setting? Have you had multiple hospitalizations? Have you been a patient in a skilled nursing facility? Have you received any antibiotic in the last 3 months? In light of the potentially severe consequences of hardware infection and the fact 
that MRSA screening is relatively inexpensive and easy to perform, we believe that it is worthwhile to pursue such screening.

\section{Intraoperative Impedance Checks}

Impedance checks can be used to verify the appropriate overlapping of contacts and to identify fractures in the lead extension system. We now check impedance 1) after securing all connections with the torque wrench to verify appropriate connectivity; 2) after placing and tucking all hardware pieces in their respective pockets to verify that the hardware was not injured by manipulation; and 3) after final closure to verify no nicking by sutures. We have found abnormal impedance in a number of extension wires at the second check after finding normal impedance at the first check and were able to replace these extensions before closure.

\section{Seizure}

The mechanism of seizure can be venous congestion at the cortex, which can be a consequence of electrocautery. Like others, we believe that it is necessary to open the pia before introducing the cannula (personal communication, K. Foote, June 6, 2012). We have been able to successfully minimize pneumocephalus by opening the dura with monopolar electrocautery and a sharp tip stylet. We then insert the stylet into the pia to create an opening without the use of electrocautery. Fibrin glue is placed around the cannula to prevent cerebrospinal fluid loss and subsequent brain shift before lead placement. Since adopting this technique, we seldom directly visualize the brain. Instead, key steps are the use of preoperative MRI with contrast, trajectory selection that avoids sulci or blood vessels, and strict adherence to the trajectory coordinates on the frame (that is, the ring and arc coordinates on the Leksell frame) to allow for "blind" penetration of the brain in a safe manner.

To account for gravitational shift, we also maintain the same patient positioning (that is, the patient supine with the head of the bed flat) during MRI and CT scanning and throughout the procedure for asleep surgeries. Our asleep placement protocol transitioned from frameless to framebased placement in January 2013, when we began keeping the head of the bed at $0^{\circ}$ for MRI and CT scanning and for the duration of the procedure. After this transition, significant improvement in accuracy was demonstrated in a recent multivariate analysis. ${ }^{25}$ Increasingly, for our awake cases, the head of the bed is also kept flat for microelectrode recording and lead placement, and the head angle is adjusted upward only for test stimulation.

\section{Study Limitations}

There are several limitations to this study, including its retrospective nature and relatively short duration of follow-up. The mean follow-up in recently published large DBS studies of adverse events has ranged from 1 to 49.3 months. Our mean follow-up was $21.4 \pm 9.8$ months. Longer-term follow-up would have allowed us to capture more adverse events, potentially increasing the likelihood of detecting a significant difference among groups. Nevertheless, because of our small sample size and the low complication rates in each adverse event category, the detec- tion of statistically significant differences among groups is unlikely. The ability to perform additional multivariate analysis beyond what was included is also limited. Twentyfour leads were associated with the most common complication (mental status change). As shown in Table 2, the $\mathrm{p}$ value associated with this comparison is 0.29 , equating to an achieved power of $24 \%$. Given the same proportions, a sample size of 3117-more than 6 times the size of our sample-would be required to achieve significance. Similarly, the $\mathrm{p}$ value associated with seizures was 0.23 , with an achieved power of $20 \%$. Given these same proportions, 2490 patients would be required to achieve $80 \%$ power.

Another possible limitation is our restricted ability to capture accurate readmission rates. We were able to access only the 30-day readmission data for patients who were readmitted either to our institution or to other hospitals that sent their medical records to our offices. There may have been patients readmitted to other hospitals about whom we were never notified, such as patients referred from out of town, although we suspect that this number would be quite small since most of our patients reside locally.

A further limitation is the slight variability in patient characteristics between and within the awake and asleep groups. There were a significantly higher proportion of essential tremor cases in the awake versus the asleep group and a significantly greater number of GPi leads in the asleep versus the awake group. The asleep group had a greater number of bilateral cases and all-in-one cases. The remaining variables were not significantly different between and within the awake and asleep groups. We recognize that these few variations are potential confounders to the analysis; however, we do not believe that they significantly affect our ability to compare the awake and asleep groups. Because of the overall low rate of complications, we analyzed the cohort as a whole to capture all the potential adverse events.

The generalizability of our results may be limited since our specific institutional protocols for awake versus asleep DBS surgery, generator placement, and preoperative MRSA screening may not be standard at other institutions. Furthermore, given the overall low rate of complications captured and the power limitations with regard to sample size, these results may not be readily generalizable. Nevertheless, as asleep DBS surgery becomes more common, this study will provide important initial data on the rates of adverse events, LOS, and postoperative readmissions rates in a relatively large series of patients undergoing DBS surgery with general anesthesia compared with data from patients undergoing traditional awake DBS surgery with microelectrode recording.

\section{Conclusions}

Both awake and asleep DBS surgery can be performed safely with a low rate of complications. LOS and 30-day readmission rates are low for both groups. In our initial experience, asleep surgery versus traditional awake surgery probably confers no difference in the incidence of complications, LOS, or 30-day readmissions. Patients in the asleep group who were 65 years old or older may have had an increased likelihood of postoperative mental status change due to the effects of general anesthesia. 


\section{References}

1. Alexander E III, Kooy HM, van Herk M, Schwartz M, Barnes PD, Tarbell N, et al: Magnetic resonance image-directed stereotactic neurosurgery: use of image fusion with computerized tomography to enhance spatial accuracy. J Neurosurg 83:271-276, 1995

2. Aviles-Olmos I, Kefalopoulou Z, Tripoliti E, Candelario J, Akram H, Martinez-Torres I, et al: Long-term outcome of subthalamic nucleus deep brain stimulation for Parkinson's disease using an MRI-guided and MRI-verified approach. J Neurol Neurosurg Psychiatry 85:1419-1425, 2014

3. Beric A, Kelly PJ, Rezai A, Sterio D, Mogilner A, Zonenshayn M, et al: Complications of deep brain stimulation surgery. Stereotact Funct Neurosurg 77:73-78, 2001

4. Bohl MA, Ahmad S, Jahnke H, Shepherd D, Knecht L, White WL, et al: Delayed hyponatremia is the most common cause of 30-day unplanned readmission after transsphenoidal surgery for pituitary tumors. Neurosurgery 78:84-90, 2016

5. Burchiel KJ, McCartney S, Lee A, Raslan AM: Accuracy of deep brain stimulation electrode placement using intraoperative computed tomography without microelectrode recording. J Neurosurg 119:301-306, 2013

6. Burdick AP, Fernandez HH, Okun MS, Chi YY, Jacobson C, Foote KD: Relationship between higher rates of adverse events in deep brain stimulation using standardized prospective recording and patient outcomes. Neurosurg Focus 29(2):E4, 2010

7. Carlson JD, Neumiller JJ, Swain LD, Mark J, McLeod P, Hirschauer J: Postoperative delirium in Parkinson's disease patients following deep brain stimulation surgery. J Clin Neurosci 21:1192-1195, 2014

8. Chen T, Mirzadeh Z, Chapple K, Lambert M, Dhall R, Ponce FA: "Asleep" deep brain stimulation for essential tremor. J Neurosurg 124:1842-1849, 2016

9. Constantoyannis C, Berk C, Honey CR, Mendez I, Brownstone RM: Reducing hardware-related complications of deep brain stimulation. Can J Neurol Sci 32:194-200, 2005

10. DeLong MR, Huang KT, Gallis J, Lokhnygina Y, Parente B, Hickey P, et al: Effect of advancing age on outcomes of deep brain stimulation for Parkinson disease. JAMA Neurol 71:1290-1295, 2014

11. Fenoy AJ, Simpson RK Jr: Management of device-related wound complications in deep brain stimulation surgery. $\mathbf{J}$ Neurosurg 116:1324-1332, 2012

12. Fenoy AJ, Simpson RK Jr: Risks of common complications in deep brain stimulation surgery: management and avoidance. J Neurosurg 120:132-139, 2014

13. Goodman RR, Kim B, McClelland S III, Senatus PB, Winfield LM, Pullman SL, et al: Operative techniques and morbidity with subthalamic nucleus deep brain stimulation in 100 consecutive patients with advanced Parkinson's disease. J Neurol Neurosurg Psychiatry 77:12-17, 2006

14. Greenberg BD, Malone DA, Friehs GM, Rezai AR, Kubu CS, Malloy PF, et al: Three-year outcomes in deep brain stimulation for highly resistant obsessive-compulsive disorder. Neuropsychopharmacology 31:2384-2393, 2006

15. Hamani C, Lozano AM: Hardware-related complications of deep brain stimulation: a review of the published literature. Stereotact Funct Neurosurg 84:248-251, 2006

16. Hariz MI, Rehncrona S, Quinn NP, Speelman JD, Wensing C: Multicenter study on deep brain stimulation in Parkinson's disease: an independent assessment of reported adverse events at 4 years. Mov Disord 23:416-421, 2008

17. Holtzheimer PE, Mayberg HS: Neuromodulation for treatment-resistant depression. F1000 Med Rep 4:22, 2012

18. Jacob RL, Geddes J, McCartney S, Burchiel KJ: Cost analysis of awake versus asleep deep brain stimulation: a single academic health center experience. J Neurosurg 124:15171523,2016
19. Joint C, Nandi D, Parkin S, Gregory R, Aziz T: Hardwarerelated problems of deep brain stimulation. Mov Disord 17 (Suppl 3):S175-S180, 2002

20. Kondziolka D, Whiting D, Germanwala A, Oh M: Hardwarerelated complications after placement of thalamic deep brain stimulator systems. Stereotact Funct Neurosurg 79:228233, 2002

21. Kooy HM, van Herk M, Barnes PD, Alexander E III, Dunbar $\mathrm{SF}$, Tarbell NJ, et al: Image fusion for stereotactic radiotherapy and radiosurgery treatment planning. Int J Radiat Oncol Biol Phys 28:1229-1234, 1994

22. Kupsch A, Benecke R, Müller J, Trottenberg T, Schneider $\mathrm{GH}$, Poewe W, et al: Pallidal deep-brain stimulation in primary generalized or segmental dystonia. N Engl J Med 355:1978-1990, 2006

23. Laxton AW, Tang-Wai DF, McAndrews MP, Zumsteg D, Wennberg R, Keren R, et al: A phase I trial of deep brain stimulation of memory circuits in Alzheimer's disease. Ann Neurol 68:521-534, 2010

24. Mikos A, Pavon J, Bowers D, Foote KD, Resnick AS, Fernandez $\mathrm{HH}$, et al: Factors related to extended hospital stays following deep brain stimulation for Parkinson's disease. Parkinsonism Relat Disord 16:324-328, 2010

25. Mirzadeh Z, Chapple K, Lambert M, Dhall R, Ponce FA: Stereotactic technique determines accuracy and efficiency in "asleep" DBS. Stereotact Funct Neurosurg 92 (Suppl 1):36, 2014 (Abstract \#148)

26. Mirzadeh Z, Chapple K, Lambert M, Dhall R, Ponce FA: Validation of CT-MRI fusion for intraoperative assessment of stereotactic accuracy in DBS surgery. Mov Disord 29:17881795,2014

27. Mirzadeh Z, Chapple K, Lambert M, Evidente VG, Mahant P, Ospina MC, et al: Parkinson's disease outcomes after intraoperative CT-guided "asleep" deep brain stimulation in the globus pallidus internus. J Neurosurg 124:902-907, 2016

28. Moghavem N, Morrison D, Ratliff JK, Hernandez-Boussard T: Cranial neurosurgical 30-day readmissions by clinical indication. J Neurosurg 123:189-197, 2015

29. Oh MY, Abosch A, Kim SH, Lang AE, Lozano AM: Longterm hardware-related complications of deep brain stimulation. Neurosurgery 50:1268-1276, 2002

30. Ostrem JL, Galifianakis NB, Markun LC, Grace JK, Martin AJ, Starr PA, et al: Clinical outcomes of PD patients having bilateral STN DBS using high-field interventional MR-imaging for lead placement. Clin Neurol Neurosurg 115:708-712, 2013

31. Ostrem JL, Ziman N, Galifianakis NB, Starr PA, Luciano MS, Katz M, et al: Clinical outcomes using ClearPoint interventional MRI for deep brain stimulation lead placement in Parkinson's disease. J Neurosurg 124:908-916, 2016

32. Patel DM, Walker HC, Brooks R, Omar N, Ditty B, Guthrie BL: Adverse events associated with deep brain stimulation for movement disorders: analysis of 510 consecutive cases. Neurosurgery 11 (Suppl 2):190-199, 2015

33. Pepper J, Zrinzo L, Mirza B, Foltynie T, Limousin P, Hariz $\mathrm{M}$ : The risk of hardware infection in deep brain stimulation surgery is greater at impulse generator replacement than at the primary procedure. Stereotact Funct Neurosurg 91:5665, 2013

34. Pezeshkian P, DeSalles AA, Gorgulho A, Behnke E, McArthur D, Bari A: Accuracy of frame-based stereotactic magnetic resonance imaging vs frame-based stereotactic head computed tomography fused with recent magnetic resonance imaging for postimplantation deep brain stimulator lead localization. Neurosurgery 69:1299-1306, 2011

35. Pollak P, Fraix V, Krack P, Moro E, Mendes A, Chabardes S, et al: Treatment results: Parkinson's disease. Mov Disord 17 (Suppl 3):S75-S83, 2002

36. Ponce FA, Lozano AM: Deep brain stimulation state of the 
art and novel stimulation targets. Prog Brain Res 184:311324,2010

37. Schuurman PR, Bosch DA, Bossuyt PM, Bonsel GJ, van Someren EJ, de Bie RM, et al: A comparison of continuous thalamic stimulation and thalamotomy for suppression of severe tremor. N Engl J Med 342:461-468, 2000

38. Sillay KA, Larson PS, Starr PA: Deep brain stimulator hardware-related infections: incidence and management in a large series. Neurosurgery 62:360-367, 2008

39. Starr PA, Markun LC, Larson PS, Volz MM, Martin AJ, Ostrem JL: Interventional MRI-guided deep brain stimulation in pediatric dystonia: first experience with the ClearPoint system. J Neurosurg Pediatr 14:400-408, 2014

40. Starr PA, Martin AJ, Ostrem JL, Talke P, Levesque N, Larson PS: Subthalamic nucleus deep brain stimulator placement using high-field interventional magnetic resonance imaging and a skull-mounted aiming device: technique and application accuracy. J Neurosurg 112:479-490, 2010

41. Verla T, Marky A, Farber H, Petraglia FW III, Gallis J, Lokhnygina Y, et al: Impact of advancing age on postoperative complications of deep brain stimulation surgery for essential tremor. J Clin Neurosci 22:872-876, 2015

42. Videnovic A, Metman LV: Deep brain stimulation for Parkinson's disease: prevalence of adverse events and need for standardized reporting. Mov Disord 23:343-349, 2008

43. Voges J, Hilker R, Bötzel K, Kiening KL, Kloss M, Kupsch A, et al: Thirty days complication rate following surgery performed for deep-brain-stimulation. Mov Disord 22:14861489,2007
44. Weaver FM, Follett K, Stern M, Hur K, Harris C, Marks WJ $\mathrm{Jr}$, et al: Bilateral deep brain stimulation vs best medical therapy for patients with advanced Parkinson disease: a randomized controlled trial. JAMA 301:63-73, 2009

45. Zrinzo L, Foltynie T, Limousin P, Hariz MI: Reducing hemorrhagic complications in functional neurosurgery: a large case series and systematic literature review. J Neurosurg 116:84-94, 2012

\section{Disclosures}

Dr. Ponce is a consultant for Medtronic, plc, and has received financial support from the Barrow Center for Neuromodulation.

\section{Author Contributions}

Conception and design: Ponce, Chen, Mirzadeh. Acquisition of data: Ponce, Chen, Lambert. Analysis and interpretation of data: Chen. Drafting the article: Ponce, Chen. Critically revising the article: Ponce. Reviewed submitted version of manuscript: Ponce. Statistical analysis: Chen, Chapple. Administrative/technical/ material support: Lambert. Study supervision: Ponce.

\section{Correspondence}

Francisco A. Ponce, c/o Neuroscience Publications, Barrow Neurological Institute, St. Joseph's Hospital and Medical Center, 350 W Thomas Rd., Phoenix, AZ 85013.email: neuropub@ dignityhealth.org. 